\title{
BMJ Open Effectiveness of combined eccentric and concentric exercise over traditional cardiac exercise rehabilitation programme in patients with chronic heart failure: protocol for a randomised controlled study
}

\author{
Guillaume Plaquevent-Hostache, ${ }^{1}$ Julianne Touron, ${ }^{2}$ Frédéric Costes, ${ }^{2,3}$ \\ Hélène Perrault, ${ }^{4}$ Guillaume Clerfond, ${ }^{5}$ Christine Cuenin, ${ }^{1}$ Andreea Moisa, ${ }^{1}$ \\ Bruno Pereira, ${ }^{6}$ Marie-Claire Boiteux, ${ }^{1}$ Romain Eschalier, ${ }^{5}$ Ruddy Richard ${ }^{2,3}$
}

To cite: Plaquevent-Hostache $\mathrm{G}$ Touron J, Costes F, et al. Effectiveness of combined eccentric and concentric exercise over traditional cardiac exercise rehabilitation programme in patients with chronic heart failure: protocol for a randomised controlled study. BMJ Open 2019;9:e028749. doi:10.1136/ bmjopen-2018-028749

- Prepublication history for this paper is available online. To view these files, please visit the journal online (http://dx.doi. org/10.1136/bmjopen-2018028749).

GP-H and JT contributed equally.

Received 21 December 2018 Revised 19 August 2019 Accepted 28 August 2019

Check for updates

(c) Author(s) (or their employer(s)) 2019. Re-use permitted under CC BY-NC. No commercial re-use. See rights and permissions. Published by BMJ.

For numbered affiliations see end of article.

Correspondence to Professor Ruddy Richard; ruddy.richard@uca.fr

\section{ABSTRACT}

Introduction Exercise-based rehabilitation is a standard feature of chronic heart failure management. The effectiveness of eccentric exercise could offer new opportunities for better tailoring rehabilitation programme to patients' limitations. The goal of the study is to contrast the impact of a mixed eccentric and concentric cycling training programme, to that of conventional concentric cycling rehabilitation in patients with chronic heart failure (peak oxygen consumption $\left(\mathrm{VO}_{2 \text { Peak }}\right)<15 \mathrm{~mL} \cdot \mathrm{kg}^{-1} \cdot \mathrm{min}^{-1}$, ejection fraction $<40 \%$ ).

Methods and analysis it is a prospective, open, controlled and randomised study $(2 \times 25$ subjects) carried out in a single centre. Subjects will perform five exercise sessions per week per the randomisation outcome, with the intervention group performing eccentric in three of the five weekly sessions while the control group will perform the five sessions of concentric exercise. Cycling intensity will be the same in both groups and fixed to the power associated with the first ventilatory threshold. Self-management education programme, callisthenics sessions and muscle strength trainings will also be carried out as for any heart failure patient normally included in the rehabilitation programme. The primary outcome will be the change in distance covered during the 6 min walk test. Secondary outcomes will include other physical mobility parameters, functional exercise capacities, quality of life and body composition as well as skeletal muscle properties including mitochondrial function parameters.

Ethics and dissemination The study has been approved by the institutional ethics review board (17.079) and the French regulatory authority for research (2017-A0096944). Adverse events that could occur during the protocol will be reported to the principal investigator. The results will be published in an international peer-reviewed journal.

Trial registration number NCT03716778.
Strengths and limitations of this study

This is a monocentric randomised controlled trial.

- The trial will include 50 chronic heart failure patients with a reduced ejection fraction $(<40 \%)$ referred for a 5 week cardiac rehabilitation programme.

- The trial will assess the relevance of a new combined training strategy (ie, concentric and eccentric cycling) for a more effective patient rehabilitation.

- Functionality, aerobic capacity and skeletal muscle parameters obtained through biopsy sample, will be assessed before and after the training period, which will allow between groups and individual comparisons.

There is no assessor or patient blinding.

\section{INTRODUCTION}

Over the last decade, consensus has been reached by Heart Failure Associations Expert groups for a grade IA level recommendation regarding regular and structured physical conditioning in the management of chronic heart failure (CHF). ${ }^{12}$ The strong recommendation is based on a growing set of experimental evidence showing improved exercise capacity, quality of life and a potential reduction in mortality after exercise training. Yet despite many clinical trials carried out, the ideal exercise rehabilitation in the standard management of CHF still does not exist. The reduced ability of CHF patients to sustain the circulatory and metabolic requirements of the prescribed mechanical power outputs is well recognised and may be an important contributor to the potential suboptimal use and effectiveness of exercise rehabilitation. ${ }^{3}$ In these patients, the major functional 
exercise limitation is of a central nature and relates to the extent of their cardio-circulatory impairment rather than to peripheral muscle capacity. ${ }^{4}$ It follows that the exercise-training enhancement capability will depend on the overall effectiveness of oxygen delivery to muscles and not on the ability of the latter to contract.

Over the past 10 years, there has also been a growing interest for the use of eccentric exercise as rehabilitation modality of interest for individuals suffering from chronic cardiorespiratory impairments on account of its lower metabolic, ventilatory and circulatory demands for any given external power output compared with conventional concentric exercise. ${ }^{5-7}$ Eccentric contraction is characterised by the ability to generate work during the lengthening of the muscle (contraction during the stretching phase) resulting in amplification of the biological adaptive stimulus owing to the additive influence of the mechanical loads being applied. ${ }^{89}$ So far, the eccentric exercise mode has been mainly applied to isolated segmental muscle strength trainings, but with the new availability on the market of eccentric cycle ergometers specifically designed for dynamic eccentric cycling, training protocols of 'moderate load eccentric exercise' are being increasingly used in populations with reduced physical capacities. ${ }^{10}$ This approach allows subjects to achieve a mechanical power overload three times that performed in concentric but for the same metabolic stimulation. ${ }^{11-13}$

In most cases, studies are designed to assess the effects of dynamic eccentric exercise as compared with those of conventional concentric training programme. ${ }^{14-16}$ The subjects are randomised in comparative groups (eccentric vs concentric) and results of physical fitness and functional capacity are evaluated before and after performing exercise training sessions of the same duration, at a percentage of the peak oxygen consumption $\left(\mathrm{VO}_{2 \text { Peak }}\right)$ or maximal heart rate determined from an incremental concentric exercise test. At the end of the training programme, usually 6-8weeks (three sessions of 30-45 min per week), the effects are compared with differences mainly related to the increase in muscle mass and strength in eccentric groups. ${ }^{1417}$

An alternative approach could be, not to contrast the impact of concentric and eccentric exercise training, but to enhance the effect through the addition of a concurrent eccentric exercise programme. Because higher cycling loads, or mechanical power output, can be sustained using the eccentric modality, one might anticipate that the combination of eccentric and concentric exercise training could serve to potentiate the beneficial effects of each modality performed separately. In other words, 'the whole being greater than the sum of the parts'.

The goal of the study is thus to compare the impact of a mixed rehabilitation programme (ie, eccentric in addition to conventional concentric cycling, labelled ECC) to that of a conventional cycling rehabilitation programme alone (labelled CON) in CHF patients admitted to a clinical rehabilitation centre.

\section{METHODS}

The study is a prospective, open, controlled study in two parallel groups carried out in a single rehabilitation centre (ie, Medical Clinic of Cardio-Pneumology of Durtol, France) (table 1). The subjects, if they meet the inclusion criteria, will be randomised into two groups (ECC and $\mathrm{CON}$ ). Stratification will be performed according to sex and pathology: ischaemic versus non-ischaemic CHF patients. The medical file of each patient addressed to the rehabilitation centre will be screened. Patients will be included in the study: (1) on referral to the cardiac rehabilitation centre to take part in the structured 5-week exercise training programme, (2) if they meet clinical inclusion criteria and (3) if they provide their signed consent.

\section{Study population}

Inclusion criteria

- CHF patient with reduced ejection fraction (EF $<40 \%)$.

- Aged 45-75 years.

- Referred for participation in the regular 5-week structured rehabilitation programme.

- $\mathrm{VO}_{2 \mathrm{Peak}}<15 \mathrm{~mL} \cdot \mathrm{kg}^{-1} \cdot \mathrm{min}^{-1}$.

- At distance from an acute coronary syndrome or myocarditis (at least 3 months) and clinically stabilised.

- Beneficiary or affiliated with social security coverage.

\section{Exclusion criteria}

- Contraindications for cardiac exercise rehabilitation as per consensus guidelines and recommendations.

- Non-stabilised acute coronary syndrome.

- Current acute heart failure.

- NYHA (New York Heart Association) class IV.

- Patient having sustained a recent procedure that could act as a confounder to increase EF or performance on the 6 min walking distance (ie, coronary artery bypass graft surgery, percutaneous coronary intervention, cardiac resynchronisation therapy, valvular surgery or reparation).

- Severe, uncontrolled disturbances in ventricular rhythm.

- Intracardiac thrombus with high risk of embolism.

- Pericardial effusion of medium to high importance.

- Recent history of thrombophlebitis with or without pulmonary embolism.

- Obstacle to severe and/or symptomatic left ventricular ejection.

- Any progressive inflammatory and/or infectious condition.

- Severe and symptomatic pulmonary arterial hypertension.

- Inability to perform physical activity.

- Reversible heart disease.

- Patients undergoing circulatory assistance.

- Patients taking blood thinner medication.

- Patients with known allergy to Xylocaine.

- Pregnant or breastfeeding women. 
Table 1 WHO trial registration data set summary

\begin{tabular}{|c|c|}
\hline Data category & Information \\
\hline $\begin{array}{l}\text { Primary registry and trial identifying } \\
\text { number }\end{array}$ & ClinicalTrials.gov NCT03716778 \\
\hline Date of registration in primary registry & 23 October 2018 \\
\hline Secondary identifying numbers & 2017-A00969-44 (ANSM) \\
\hline $\begin{array}{l}\text { Source(s) of monetary or material } \\
\text { support }\end{array}$ & $\begin{array}{l}\text { University Hospital of Clermont-Ferrand } \\
\text { Medical Clinic of Cardio-Pneumology of Durtol }\end{array}$ \\
\hline Secondary sponsor(s) & Medical Clinic of Cardio-Pneumology of Durtol \\
\hline Contact for public queries & Lise LACLAUTRE: drci@chu-clermontferrand.fr \\
\hline Contact for scientific queries & Pr. Ruddy RICHARD (MD, PhD): ruddy.richard@uca.fr \\
\hline Public title & Rehabilitation by Eccentric eXercise in Heart Failure patients (REX-HF) \\
\hline Scientific title & $\begin{array}{l}\text { Effectiveness of combined eccentric and concentric exercise over traditional cardiac exercise } \\
\text { rehabilitation programme in patients with chronic heart failure: a randomised controlled study }\end{array}$ \\
\hline Intervention(s) & $\begin{array}{l}\text { Control group: usual medical care according to the rehabilitation recommendations } \\
\text { Experimental group: mixed programme combining eccentric pedalling sessions with the usual sessions }\end{array}$ \\
\hline Key inclusion and exclusion criteria & $\begin{array}{l}\text { Ages eligible for study: } 18<\text { age }<75 \text {; Sexes eligible for study: both; Accepts healthy volunteers: no } \\
\text { Inclusion criteria: Patient with systolic chronic heart failure, referred for participation in the } 5 \text {-week } \\
\text { rehabilitation programme, at a time distance from an acute coronary syndrome or myocarditis (less than } \\
3 \text { months old) and clinically stabilised, beneficiary or affiliated with social security coverage } \\
\text { Exclusion criteria: all the usual criteria considered contra-indicated for cardiac exercise rehabilitation } \\
\text { according to the consensus recommendations, non-stabilised acute coronary syndrome, } \\
\text { decompensated heart insufficiency, stage IV NYHA, severe, uncontrolled disturbances in ventricular } \\
\text { rhythm, presence of an intracardiac thrombus with high risk of embolism, presence of pericardial } \\
\text { effusion of medium to high importance, recent history of thrombophlebitis with or without pulmonary } \\
\text { embolism, obstacle to severe and/or symptomatic left ventricular ejection, any progressive } \\
\text { inflammatory and/or infectious condition, severe and symptomatic pulmonary arterial hypertension, } \\
\text { inability to perform physical activity, reversible heart disease, patients undergoing circulatory } \\
\text { assistance, patients taking blood thinner medication, patients with known allergy to Xylocaine, pregnant } \\
\text { or lactating women, women of childbearing age without contraception, patient under tutorship, } \\
\text { curatorship or deprived of liberty }\end{array}$ \\
\hline Date of first enrolment & November 2018 \\
\hline Target sample size & 50 \\
\hline Recruitment status & Recruiting \\
\hline Primary outcome(s) & 6 -min walk test \\
\hline Key secondary outcomes & $\begin{array}{l}\text { Time up and go test } \\
\text { Quadriceps isometric muscular strength } \\
\text { Peak } \mathrm{VO}_{2} \\
\text { Lean and fat body mass }\end{array}$ \\
\hline
\end{tabular}

NYHA, New York Heart Association.

- Childbearing age women without contraception.

- Patient under tutorship, curatorship or deprived of liberty.

\section{Study design and patient management}

Patients will be followed over the period of 5 weeks (ie, 20 exercise sessions) covering the duration of the standard structured cardiac rehabilitation programme in France and covered by the French healthcare insurance programme (figure 1). ${ }^{18}$ They will undergo a standard evaluation as any CHF patient referred for rehabilitation including an initial 12-lead ECG, a trans-thoracic echocardiography, and a cardiopulmonary exercise test (CPET) with continuous gas exchange analysis and non-invasive measurement of cardiac output. A standard biological blood analysis will be performed for complete blood count, blood electrolytes, C-reactive protein, N-terminal 


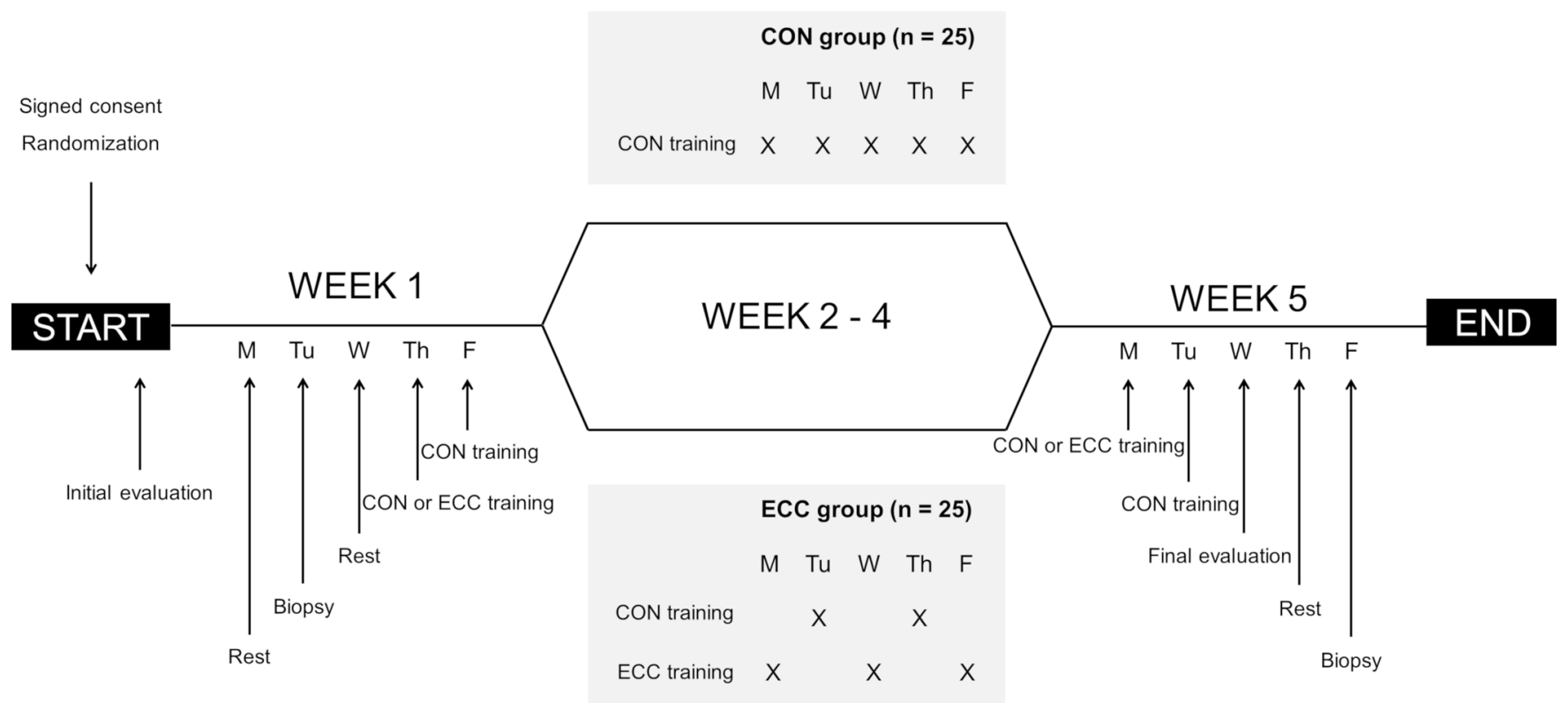

Figure 1 Study design of the 5-week rehabilitation programme. CON, concentric; ECC, eccentric; M, Monday; Tu, Tuesday; W, Wednesday; Th, Thursday; F, Friday.

prohormone of brain natriuretic peptide and nutritional status with albumin and prealbumin. Subjects will also perform a 6-min walk test (6MWT), a time up and go test (TUGT), an isometric muscular strength (IMS) measurement, a gait speed test and an endurance cycling test. Body composition will be measured and patients will be asked to complete short form health survey (SF36). Finally, a muscle biopsy of the Vastus Lateralis will be realised using the modified Bergström method. All initial tests and evaluations will be repeated at the end of the 5-week exercise rehabilitation programme, to compare the training-induced adaptations.

\section{Exercise training approach}

All subjects will perform five supervised cycle ergometer training sessions per week. Each 30-min session will be achieved at an intensity corresponding to the predetermined first ventilator threshold $\left(\mathrm{VT}_{1}\right)$ with a pedalling rate of 60 rotations per minute (RPM). As per randomisation outcome, the ECC group will perform three of the five weekly sessions in eccentric and the two others in concentric, while the CON group will perform all five weekly sessions using the concentric modality. The training programme is designed such that the total amount of work carried out on the ergometers will be equivalent (iso-energetic) for both groups (Work $=[\mathrm{Ex}-$ ercise Time $(\mathrm{min}) \times$ Oxygen Consumption $\left(\mathrm{L} \cdot \mathrm{min}^{-1}\right) \times \mathrm{O}_{2}$ energy equivalent coefficient $\left.\left.\left(\mathrm{kJ} \cdot \mathrm{LO}_{2}^{-1}\right)\right]\right)$. The ECC group will be submitted to a habituation period of five sessions during which duration and intensity will be progressively increased. The same progression will be applied over the same period in the CON group. In all cases, the training sessions will be adjusted to the subjects' tolerance. Sessions will be performed under electrocardiographic control and blood pressure monitoring as per the usual practice.
Self-management education programme, callisthenics sessions and muscle strength training will also be carried out as included in the structured rehabilitation programme (ie, gymnastics every day and muscular reinforcement three times a week).

\section{Eccentric exercise program}

Eccentric cycling will be conducted at an intensity corresponding to $\mathrm{VT}_{1}$ requirement using commercially available eccentric motor-driven ergometer (Cyclus2 Eccentric Recumbent, RBM Elektronik-automation, MSE Medical, Duttlenheim, France). This device works as follows: a motor drives the pedals in a backward rotation and subjects are asked to resist the rotary motion by applying force, resulting in eccentric muscle contractions of the extensor muscles.

Intensity and duration will be progressively increased during the first five sessions in order to minimise the extent of exercise-induced muscle soreness associated with unaccustomed eccentric exercise. ${ }^{5}{ }^{12}$ Patients will be instructed to maintain a cycling rate of 60RPM. The target duration after the habituation period will be $30 \mathrm{~min}$. A typical eccentric habituation pattern is shown in table 2. During habituation period, session's duration and workload will not be increased if participants suffer

\begin{tabular}{|c|c|c|c|}
\hline $\begin{array}{l}\text { Session of } \\
\text { habituation }\end{array}$ & $\begin{array}{l}\text { Eccentric } \\
\text { power (W) }\end{array}$ & $\begin{array}{l}\text { Concentric power } \\
\text { equivalent (W) }\end{array}$ & $\begin{array}{l}\text { Duration } \\
\text { (min) }\end{array}$ \\
\hline Session 1 & 50 & 16 & 10 \\
\hline Session 2 & 75 & 25 & 15 \\
\hline Session 3 & 100 & 33 & 20 \\
\hline Session 4 & 125 & 40 & 25 \\
\hline Session 5 & 150 & 50 & 30 \\
\hline
\end{tabular}


from muscle soreness, as reported by a score above 3 on a visual analogic scale (0-10 scale) or when the rated perceived exertion exceeds 13 on the 6-20 Borg scale during the exercise session.

\section{Primary end point}

The primary end-point criterion will be the change in $6 \mathrm{MWT}$ distance covered, expressed as the percentage of change between the test performance at the end of the rehabilitation programme, and that of the initial test. Taking as reference point previous exercise rehabilitation studies in patients with chronic disease, the expected gain in walking distance would be at least of $5 \% .{ }^{19-21}$ Our expectation is that a significant greater increase in $6 \mathrm{MWT}$ distance will be seen in the group having completed the mixed exercise protocol (ECC) as compared with CON group.

\section{Secondary end points}

The secondary study criteria are related to prerehabilitation and postrehabilitation parameters values as follows:

- Clinical evaluation of physical mobility:

- TUGT.

- Quadriceps IMS.

- Gait speed test.

- CPET results:

- $\mathrm{VO}_{2 \text { Peak }}$.

- Peak power $\left(\mathrm{P}_{\text {Peak }}\right)$.

- Peak cardiac output $\left(Q_{\text {Peak }}\right)$.

- Values of oxygen consumption $\left(\mathrm{VO}_{2}\right)$, power $(\mathrm{P})$ and cardiac output $(\mathrm{Q})$ at $\mathrm{VT}_{1}\left(\mathrm{ie}, \mathrm{VO}_{2 \mathrm{VT} 1} ; \mathrm{P}_{\mathrm{VT} 1}\right.$; $\left.\mathrm{Q}_{\mathrm{VT} 1}\right)$.

- Values of minute ventilation (VE) and carbon dioxide production $\left(\mathrm{VCO}_{2}\right)$ to calculate $\mathrm{VE} / \mathrm{VO}_{2}$ and $\mathrm{VE} / \mathrm{VCO}_{2}$ at $\mathrm{VT}_{1}$ and at peak exercise.

- Time to exhaustion during the endurance cycling exercise test.

- NYHA functional score.

- SF36 scores.

- Biological parameters including albumin, prealbumin and BNP.

- Body composition (ie, lean and fat mass) evaluated by Dual-energy X-ray absorptiometry (DEXA).

- Muscle biopsy analysis.

\section{Measurements}

Six-minute walk test

Patients will be asked to cover the longest distance over a $30 \mathrm{~m}$ mark in a period of $6 \mathrm{~min}$ with or without stopping and with standardised encouragements according to standard recommendations, the first test being performed twice. ${ }^{22}$ Pulsed oxygen saturation and heart rate will be monitored continuously throughout the test using a digital oximeter.

Time up and go test

TUGT evaluates seated transfers, standing, walking and changes of direction. ${ }^{23}{ }^{24}$ The subject sits on a chair with armrests facing a wall located at a 3-m distance. The subject must stand up, walk to the wall, turn around without touching it, return to the chair, and sit down again. A score assessing assurance and movement stability on a scale from 1 to 5 will be determined by the evaluator such that:

1. No instability.

2. Very slightly abnormal (slow execution).

3. Moderately abnormal (hesitation, compensatory movements).

4. Abnormal (the patient stumbles).

5. Very abnormal (permanent risk of falling).

\section{Quadriceps isometric muscular strength}

Maximum muscle strength of the quadriceps, on the dominant limb, will be measured on a bench according to the instructions (MicroFET2, Hoggan Scientific, Salt Lake City, Utah, USA):

- Strength during isometric contraction (at $90^{\circ}$ knee flexion).

- Standardised patient's position with the arms crossed on the chest, the absence of back support during the measurement and the maintenance of the hips and the contralateral leg to avoid any compensating movement.

Three reproducible measurements $( \pm 10 \%)$ will be carried out at 1-min intervals and the highest value will be retained. ${ }^{25} 26$

\section{Gait speed test}

Evaluation of the gait speed over $4 \mathrm{~m} .{ }^{27}$ Participants will be instructed to walk from a standing start, at a normal and comfortable pace for them. Patient will walk through a $1 \mathrm{~m}$ 'acceleration' zone, a central $4 \mathrm{~m}$ 'testing' zone, and a $1 \mathrm{~m}$ 'deceleration' zone. A trained evaluator will start and stop timing respectively when the participant's foot will contact the floor at the beginning and at the end of the central testing zone. Gait speed will be calculated using distance in metres and time in seconds.

\section{Cardiopulmonary exercise test}

The patient will perform a symptom-limited incremental test on a traditional concentric cycling ergometer (VIAsprint 150P, Ergoline, Bitz, Germany) with continuous monitoring of ECG, pulsed oxygen saturation, cardiac output (Physioflow Enduro, Manatec Bio-medical, Macheren, France) and breath-by-breath expired gas analysis (MasterScreen CPX, Carefusion, Hoechberg, Germany) as per standard recommendation guidelines. ${ }^{21}$ The workload increments will be of $10 \mathrm{~W} \cdot \mathrm{min}^{-1}$. The $\mathrm{VT}_{1}$ will be determined using the V-slope method. ${ }^{28} \mathrm{VO}_{2 \text { Peak }}, \mathrm{P}_{\text {Peak }}$ and $\mathrm{Q}_{\text {Peak }}$ will be measured at the end of the last completed exercise level.

\section{Endurance cycling exercise test}

This test is performed using a conventional cycling ergometer (ER5/ES3000, MSE Medical, Duttlenheim, France) to assess the endurance capacity of patients. ${ }^{29} 30$ The subject will be asked to cycle as long as possible at a constant power output set at $\left[\left(\mathrm{P}_{\text {Peak }}+\mathrm{P}_{\mathrm{VTI}}\right) / 2\right]$, with $\mathrm{P}_{\text {Peak }}$ 
and $\mathrm{P}_{\mathrm{VT} 1}$ values predetermined from the incremental concentric cycling exercise test. Total cycling time will be measured without encouraging the subject. The 'time limit' is the time elapsed from the start of cycling to that time at which the subject can no longer maintain the requested pedalling of 50-60 RPM.

\section{Body composition}

Body composition will be assessed by DEXA (Hologic QDR-4500A, Bedford, Massachusetts, USA), since it is considered as a reference method allowing accurate measurement of body compartments. ${ }^{31}$ Measurements will focus on total body composition (more particularly total lean mass) but also segmental lean and fat mass (upper and lower limbs) and abdominal fat mass.

\section{Disease severity and quality of life}

Patients' functional status will be expressed using the NYHA classification frequently used as a reliable measure in CHF patients. ${ }^{32}$ Quality of life will be determined using the validated SF36 questionnaire. ${ }^{33}$

\section{Blood testing}

Biological assessments will be performed as part of the usually prescribed patient care. In addition, albumin and prealbumin will be measured to characterise their nutritional status.

\section{Muscle biopsy}

Muscular adaptations will be studied on Vastus Lateralis samples taken during a muscular biopsy using the modified Bergström technique. ${ }^{34}$ Some of the fresh tissue will be used to study mitochondrial functions on permeabilised muscle fibres by respirometry (Oxygraph-2k G series, Oroboros Instruments, Innsbruck, Austria) and fluorimetry (Oxygraph-2k-Fluo LED2-Module, Oroboros Instruments, Innsbruck, Austria). The rest of the biopsy will be sampled and frozen for further analysis (ie, histology, mRNA expression, biochemistry). There will be no data bank setup and the samples will only be conserved transiently for the duration of the analyses provided for in the study protocol. At the end of the study, if any samples remain, these will be destroyed. The micro-biopsy performed in the Vastus Lateralis muscle requires a 24 hours recovery and will have no impact on the exercise programme. Arm exercises will be performed to compensate during these 24 hours period in the two groups.

\section{Ethics}

All patients will receive verbal and written information on the aim of the study and the protocol. Written informed consent will be obtained prior to their inclusion in the study and before performing any specific procedure. During the study, patient will have the opportunity to ask all questions concerning the protocol to the cardiologist or investigator. They will be informed that they are free to stop the study at any time at their own discretion, in accordance with the Good Clinical Practice in current enforced under the French regulatory framework. Any adverse event that could occur during the protocol will be reported to the principal investigator. Should there be any negative impact of participating in the study on the patient's health status, the participant will be entitled to compensation in accordance with the French regulations.

Pursuant to the provisions concerning the confidentiality of data that are available to persons responsible for quality control of biomedical research, persons with direct access will take all necessary precautions to ensure the confidentiality of information (identity and patients results). Data collected will be made anonymous.

\section{Study status}

The first inclusion was scheduled in November 2018. To date, seven patients were enrolled. The recruitment period spans over 2 years with the goal to include 25 patients per treatment arm, each patient completing evaluation before and after the 5-week exercise rehabilitation programme (figure 1).

\section{Patient and public involvement}

Patients were not involved in the development and the design of the study. The burden of the intervention will not be assessed by patients themselves. Patients will receive a written summary of tests and evaluation results they will have completed during their rehabilitation and will be written informed of global study results at its end.

\section{Management of the study}

The principal investigator and the trained clinical research team will collect data. Data will be recorded for each patient in a dedicated notebook at each assessment point, and any deviation from the protocol or adverse event will be noted. Study data will also be collected and managed using REDCap (Research Electronic Data Capture) electronic data capture tools hosted at the University Hospital of Clermont-Ferrand. ${ }^{35}$ REDCap is a secure, web-based application designed to support data capture for research studies, providing:

- An intuitive interface for validated data entry.

- Audit trails for tracking data manipulation and export procedures.

- Automated export procedures for seamless data downloads to common statistical packages.

- Procedures for importing data from external sources. A clinical research assistant will be commissioned to ensure the progress of the study, the data capture according to the Standard Operating Procedures implemented at the University Hospital of Clermont-Ferrand.

\section{Statistical consideration and study size \\ Power of the study}

Sample size estimation is based on previous reports from the scientific literature, describing a SD for exercise training induced changes in the 6MWT (primary outcome) ranging between $2 \%$ and $4 \% .{ }^{19}$ For a two-sided type I error at $5 \%$ and a statistical power $(1-\beta)$ greater $80 \%$, sample size is calculated at 20 patients per arm in order to show a minimal absolute difference of $3 \%$ (more 
precisely, $\mathrm{SD}_{6 \mathrm{MWT}}=2.9$ and $1-\beta=90 \%$ ). In order to account for patients dropping out of the study, the aim is to recruit 25 patients in each randomised group for a total of 50 patients.

\section{Randomisation procedure}

A permuted-block randomisation (ie, random block sizes) will be conducted using a computer-generated random allocation (Stata statistical software, V.13, StataCorp LP, College Station, Texas, United States of America), with a 1:1 ratio allocation, ensuring complete randomness of the assignment of a patient to each randomised group. Stratification will be performed according to sex and pathology (ischaemic vs no ischaemic heart failure). To ensure that there is no bias in their selection and allocation, patients will be randomised after it is clear that they meet the inclusion criteria, that they have provided written consent and that an anonymity number has been assigned to them.

\section{Statistical analysis}

All analyses will be conducted using Stata software (V.13). A two-sided $\mathrm{p}$ value less than 0.05 will be considered to indicate statistical significance. The continuous variables will be expressed as mean and SD or as medians and IQR, according to statistical distribution. The assumption of normality will be assessed using the Shapiro-Wilk test. The categorical parameters will be expressed as the number of patients and associated percentages. The patients will be described and compared at inclusion between randomised groups according to the following variables: compliance with the eligibility criteria, epidemiological, clinical and biological characteristics and treatments.

Student's t-test or Mann-Whitney U test, if t-test's assumptions are not met (normality studied using Shapiro-Wilk test and homoscedasticity verified by Fisher-Snedecor test), will be applied to compare the primary endpoint between randomised groups. The results will be described with effect-size and 95\% CI. A multivariable analysis will then be performed using multiple linear regression to take into account adjustment on possible confounders covariates selected according to clinical relevance and to univariate results. The normality of residuals will be studied using the Shapiro-Wilk test. If appropriate, a transformation of 6MWT change will be proposed to achieve Gaussian statistical distribution. Multivariable analysis results will be presented using regression coefficients and $95 \%$ CIs.

The other continuous variables will be compared similarly with the use of the unpaired t-test or the MannWhitney $U$ test when appropriate and using the same adjustment variables described above for multivariable approach. For categorical endpoints, $\chi^{2}$ or Fisher's exact tests will be used. The results will be described as absolute difference and 95\% CIs. Furthermore, the relationships between quantitative variables will be explored with correlation coefficients (Pearson or Spearman, according to statistical distribution). At last, longitudinal analyses of correlated repeated data will be performed using random-effects models. Patient will be considered as random-effect in order to take into account between and within patient variability, whereas the following fixed effects will be studied: randomisation group, time-points evaluation and their interaction.

\section{Analysis of missing outcome data}

The primary analysis will be conducted on an intention-totreat basis, with a particular focus paid on loss to follow-up. An analysis of dropouts, considered as censored data, will be proposed using the Kaplan-Meier estimation in order to (1) study how much patients stop the study and (2) to take into account the time when they will be dropped out. The comparison between randomised groups will be conducted in univariate analysis by log-rank test and for multivariable analysis by the Cox proportional regression model. Then, a sensitivity analysis will be performed and the nature of missing data will be studied (missing at random or not). According to it, the most appropriate approach to the imputation of missing data will be proposed (last observation carried forward or multiple imputations). Then, a per-protocol analysis will be also considered to evaluate the efficacy of the experimental arm.

\section{DISCUSSION}

This is the first study to examine the potential benefits of adding an eccentric exercise-training component to a regular structured cardiac exercise rehabilitation programme in CHF patients.

The positive effects of exercise rehabilitation programme for patients with CHF and chronic lung diseases have now long been established. ${ }^{136}$ The current challenge is to ensure that the recommendation is implemented and to continue to make gains in the clinical management of these patients. Within this framework, moderate load dynamic eccentric exercise presents an interesting alternative on account of its efficiency in enhancing muscle strength even in patients with severe degrees of impairment. ${ }^{7}$

The functional limitation of CHF patients can be related to both central cardiorespiratory dysfunctions and peripheral musculoskeletal weakness resulting from deconditioning. ${ }^{437}$ Inasmuch as biological adaptations to exercise are proportional to the nature and to the extent of the stimulus, it is only logical to think that combining exercise modalities known to exert different but complementary effects could be of value in the design of more effective rehabilitation programme.

As a first approach, the present study examines the impact of adding a regimen of dynamic eccentric cycling to a structured rehabilitation programme of conventional cycling. The working hypothesis is that patients having completed the combined exercise programme should show greater functional gains resulting from a synergistic effect of these stimuli. Repercussions of deconditioning 
and functional limitations of patients with chronic disease are far-reaching including enhanced comorbidity, decreased functional autonomy impacting the ability of patients to socialise as well as decreased self-efficacy and overall quality of life. ${ }^{38}$ The effectiveness of eccentric type exercise to enhance functional strength even in more severely affected CHF patients could offer new opportunities for better tailoring programme to their limitations.

\section{Author affiliations}

${ }^{1}$ Cardiology and Vascular Diseases, Medical Clinic of Cardio-Pneumology of Durtol, Durtol, France

${ }^{2}$ UMR1019 INRA-UCA, ASMS team, CRNH Auvergne, INRA Centre Auvergne Rhone Alpes, Clermont Ferrand, France

${ }^{3}$ Department of Sport Medicine and Functional Explorations, Centre Hospitalier Universitaire de Clermont-Ferrand, Clermont-Ferrand, France

${ }^{4}$ Faculty of Health Sciences, University of Ottawa, Ottawa, Ontario, Canada ${ }^{5}$ Department of Cardiology, Centre Hospitalier Universitaire de Clermont-Ferrand, Clermont-Ferrand, France

${ }^{6}$ Delegation to Clinical Research and Innovation, Centre Hospitalier Universitaire de Clermont-Ferrand, Clermont-Ferrand, France

Contributors The authors are solely responsible for the design and conduct of the study. They are also responsible for all the study analysis, the drafting and editing of manuscript and its final content. RR, GP-H, M-CB, RE, BP and JT conceived the study. RR, GP-H, M-CB, BP, JT and HP wrote the protocol and designed the study. JT and RR conceived the muscle biopsy analysis program. HP, JT and RR edited the English version of the manuscript. BP conceived the statistical analysis, and will perform randomisation and analysis. GP-H, M-CB, RR, RE, GC, CC, AM, FC will carry out the clinical study. RR and GP-H are the main investigators of the study, and will obtain informed consent.

Funding This work was supported by the University Hospital of Clermont-Ferrand (AOI Funding) and the Medical Clinic of Cardio-Pneumology of Durtol.

\section{Competing interests None declared.}

Patient consent for publication Not required.

Ethics approval According to the French regulation on clinical trials, the study has been submitted to the 'Comité de Protection des Personnes' (reference: 2017A00969-44) and to the 'Agence Nationale de Sécurité du Medicament' (the French regulatory authority for research). Approval from the Ethics Review Board is dated from 7 April 2018. Any modification in the protocol or informed consent during the study will be presented to the reference authority.

Provenance and peer review Not commissioned; externally peer reviewed.

Open access This is an open access article distributed in accordance with the Creative Commons Attribution Non Commercial (CC BY-NC 4.0) license, which permits others to distribute, remix, adapt, build upon this work non-commercially, and license their derivative works on different terms, provided the original work is properly cited, appropriate credit is given, any changes made indicated, and the use is non-commercial. See: http://creativecommons.org/licenses/by-nc/4.0/.

\section{REFERENCES}

1. Ponikowski P, Voors AA, Anker SD, et al. Esc guidelines for the diagnosis and treatment of acute and chronic heart failure: the task force for the diagnosis and treatment of acute and chronic heart failure of the European Society of cardiology (ESC). developed with the special contribution of the heart failure association (HFA) of the ESC. Eur J Heart Fail 2016;2016:891-975.

2. Piepoli MF, Conraads V, Corrà U, et al. Exercise training in heart failure: from theory to practice. A consensus document of the heart failure association and the European association for cardiovascular prevention and rehabilitation. Eur J Heart Fail 2011;13:347-57.

3. Hirai DM, Musch TI, Poole DC. Exercise training in chronic heart failure: improving skeletal muscle $\mathrm{O} 2$ transport and utilization. Am J Physiol Heart Circ Physiol 2015;309:H1419-H1439.

4. Doehner W, Frenneaux M, Anker SD. Metabolic impairment in heart failure: the myocardial and systemic perspective. J Am Coll Cardiol 2014;64:1388-400.
5. Rocha Vieira DS, Baril J, Richard R, et al. Eccentric cycle exercise in severe COPD: feasibility of application. COPD 2011;8:270-4.

6. Chasland LC, Green DJ, Maiorana AJ, et al. Eccentric cycling: a promising modality for patients with chronic heart failure. Med Sci Sports Exerc 2017;49:646-51.

7. LaStayo P, Marcus R, Dibble L, et al. Eccentric exercise in rehabilitation: safety, feasibility, and application. J Appl Physiol 2014;116:1426-34.

8. Roig M, O'Brien K, Kirk G, et al. The effects of eccentric versus concentric resistance training on muscle strength and mass in healthy adults: a systematic review with meta-analysis. $\mathrm{Br} J$ Sports Med 2009;43:556-68.

9. Franchi MV, Reeves ND, Narici MV. Skeletal muscle remodeling in response to eccentric vs. concentric loading: morphological, molecular, and metabolic adaptations. Front Physiol 2017;8.

10. Hoppeler $\mathrm{H}$. Moderate load eccentric exercise; a distinct novel training modality. Front Physiol 2016;7.

11. Perrey S, Betik A, Candau R, et al. Comparison of oxygen uptake kinetics during concentric and eccentric cycle exercise. J Appl Physiol 2001;91:2135-42.

12. Dufour SP, Lampert E, Doutreleau S, et al. Eccentric cycle exercise: training application of specific circulatory adjustments. Med Sci Sports Exerc 2004;36:1900-6.

13. Julian V, Thivel $\mathrm{D}$, Miguet $\mathrm{M}$, et al. Eccentric cycling is more efficient in reducing fat mass than concentric cycling in adolescents with obesity. Scand J Med Sci Sports 2019;29:4-15.

14. Steiner R, Meyer K, Lippuner K, et al. Eccentric endurance training in subjects with coronary artery disease: a novel exercise paradigm in cardiac rehabilitation? Eur J Appl Physiol 2004;91:572-8.

15. Meyer K, Steiner R, Lastayo P, et al. Eccentric exercise in coronary patients: central hemodynamic and metabolic responses. Med Sci Sports Exerc 2003;35:1076-82.

16. Gremeaux V, Duclay J, Deley G, et al. Does eccentric endurance training improve walking capacity in patients with coronary artery disease? A randomized controlled pilot study. Clin Rehabil 2010;24:590-9.

17. MacMillan NJ, Kapchinsky S, Konokhova Y, et al. Eccentric ergometer training promotes locomotor muscle strength but not mitochondrial adaptation in patients with severe chronic obstructive pulmonary disease. Front Physiol 2017;8.

18. Pavy $\mathrm{B}$, lliou $\mathrm{M}$, Vergès $\mathrm{B}$, et al. Référentiel des bonnes pratiques de la réadaptation cardiaque de l'adulte en 2011 2011;64.

19. Meyer K, Schwaibolda M, Westbrook S, et al. Effects of exercise training and activity restriction on 6-minute walking test performance in patients with chronic heart failure. Am Heart $J$ 1997;133:447-53.

20. Puhan MA, Mador MJ, Held U, et al. Interpretation of treatment changes in 6-minute walk distance in patients with COPD. European Respiratory Journal 2008;32:637-43.

21. Guazzi M, Dickstein K, Vicenzi M, et al. Six-minute walk test and cardiopulmonary exercise testing in patients with chronic heart failure: a comparative analysis on clinical and prognostic insights. Circ Heart Fail 2009;2:549-55.

22. ATS Committee on Proficiency Standards for Clinical Pulmonary Function Laboratories. Ats statement: guidelines for the six-minute walk test. Am J Respir Crit Care Med 2002;166:111-7.

23. Mathias S, Nayak US, Isaacs B. Balance in elderly patients: the "getup and go" test. Arch Phys Med Rehabil 1986;67:387-9.

24. Hwang R, Morris NR, Mandrusiak A, et al. Timed up and go test: a reliable and valid test in patients with chronic heart failure. $J$ Card Fail 2016;22:646-50

25. Kamiya K, Mezzani A, Hotta K, et al. Quadriceps isometric strength as a predictor of exercise capacity in coronary artery disease patients. Eur J Prev Cardiol 2014;21:1285-91.

26. Hülsmann M, Quittan M, Berger R, et al. Muscle strength as a predictor of long-term survival in severe congestive heart failure. Eur $\checkmark$ Heart Fail 2004;6:101-7.

27. Studenski S, Perera S, Patel K, et al. Gait speed and survival in older adults. JAMA 2011;305:50-8.

28. Beaver WL, Wasserman K, Whipp BJ. A new method for detecting anaerobic threshold by gas exchange. J Appl Physiol 1986;60:2020-7.

29. Andrianopoulos V, Wagers SS, Groenen MTJ, et al. Characteristics and determinants of endurance cycle ergometry and six-minute walk distance in patients with COPD. BMC Pulm Med 2014;14:97.

30. Larsen Aet al. Assessing the effect of exercise training in men with heart failure. Comparison of maximal, submaximal and endurance exercise protocols. Eur Heart J 2001;22:684-92.

31. Uszko-Lencer NHMK, Bothmer F, van Pol PEJ, et al. Measuring body composition in chronic heart failure: a comparison of methods. Eur $J$ Heart Fail 2006;8:208-14. 
32. Bennett JA, Riegel B, Bittner V, et al. Validity and reliability of the NYHA classes for measuring research outcomes in patients with cardiac disease. Heart Lung 2002;31:262-70.

33. McHorney CA, Ware JE, Rachel Lu JF, et al. The mos 36-Item short-form health survey (SF-36): III. tests of data quality, scaling assumptions, and reliability across diverse patient groups. Med Care 1994;32:40-66.

34. Shanely RA, Zwetsloot KA, Triplett NT, et al. Human skeletal muscle biopsy procedures using the modified Bergström technique. J Vis Exp 2014;(91).

35. Harris PA, Taylor R, Thielke R, et al. Research electronic data capture (REDCap)--a metadata-driven methodology and workflow process for providing translational research informatics support. J Biomed Inform 2009;42:377-81.

36. McCarthy B, Casey D, Devane D, et al. Pulmonary rehabilitation for chronic obstructive pulmonary disease. Cochrane Database Syst Rev 2015:CD003793

37. Drexler $\mathrm{H}$, Riede $\mathrm{U}$, Münzel $\mathrm{T}$, et al. Alterations of skeletal muscle in chronic heart failure. Circulation 1992;85:1751-9.

38. Yancy CW, Jessup M, Bozkurt B, et al. ACCF/AHA guideline for the management of heart failure: a report of the American College of cardiology Foundation/American heart association Task force on practice guidelines. J Am Coll Cardiol 2013;2013:e147-239. 